\title{
Using Capillary Condensation and Evaporation Isotherms to Investigate Confined Fluid Phase Behavior in Shales
}

\author{
Elizabeth Barsotti ${ }^{1, *}$, Evan Lowry ${ }^{1}$, Mohammad Piri ${ }^{1}$, and Jin-Hong Chen ${ }^{2}$ \\ ${ }^{1}$ Center of Innovation for Flow through Porous Media, Petroleum Engineering Department, University of Wyoming, Laramie, Wyoming, \\ USA \\ ${ }^{2}$ Aramco Services Company, Aramco Research Center-Houston, Houston, Texas, USA
}

\begin{abstract}
The abundance of nanopores (pores with diameters between 2 and $100 \mathrm{~nm}$ ) in shale and ultra-tight reservoirs precludes the use of common pressure-volume-temperature (PVT) analyses on reservoir fluids. The small sizes of the pores cause capillary condensation, which is a nanoconfinement-induced gas-to-liquid phase change, that can occur at pressures more than $50 \%$ below the corresponding bulk phase change of the fluid due to strong fluid-pore wall interactions. We quantify this phenomenon by measuring propane isotherms both in a synthetic nanoporous medium and a core from a shale gas reservoir. Comparison of our results in the two porous media indicates the occurrence of capillary condensation in shale rock. At the same time, we observe capillary condensation hysteresis for shale, in which the density of the fluid is significantly lighter during desorption than adsorption. This indicates structural changes to the rock matrix caused by the phase behavior of the confined fluid. We use scanning electron microscopy to corroborate our findings. These results have significant implications for determining the PVT properties, porosity, and permeability of shale and ultra-tight formations for use in reservoir modeling and production estimations.
\end{abstract}

\section{Introduction}

Current methods of shale reservoir evaluation do not account for (i) capillary condensation, a confinementinduced gas-to-liquid phase change, or (ii) continuous pore filling, a sharp increase in the density of supercritical fluids, that occur in nanopores. [1] Even though it has been shown that accounting for nanoconfinement-induced phase phenomena such as capillary condensation could increase reserves estimates by $600 \%$, [2] the current prevailing opinion only considers the existence of an adsorbed, or physisorbed, phase on the pore walls and free gas in the pore bodies. [3]

The primary reason for this is misinterpretation of isotherms measured in shale. Throughout the literature, isotherms measured for both hydrocarbons and gases, such as $\mathrm{CO}_{2}$, do not exhibit the distinct capillary condensation step defined in the IUPAC Type IV isotherm [4] or the continuous filling step that has recently been elucidated by Tan et al. [5] and Barsotti et al. [6] Instead, they appear similar to IUPAC Type I and II isotherms, [7], [8] which are attributed to mono-layer and multi-layer adsorption, respectively. [4] Therefore, they are given the same interpretation even though the IUPAC isotherms rely on fundamental assumptions about the adsorbate and adsorbent that are not true of shale rock. The Type I isotherm assumes a microporous material, while the Type II isotherm assumes a macroporous or non-porous surface. [4] Both of these cases are far removed from the wide pore size distributions, complex wettabilities, unpredictable pore geometries, and complex fluid compositions in shale. Even so, the ease with which longstanding, simplistic adsorption theories, such as Langmuir and BET, can be fit to shale adsorption isotherms has led to their widespread incorporation into shale characterization [9], [3], [10], [11] and reservoir modeling. [12]-[14]

However, we prove here that despite the fact that the shapes of shale isotherms are often similar to IUPAC isotherms, the underlying physics are generally not the same. By creating controlled pore size distributions with a synthetic nanoporous medium called MCM-41 and comparing the isotherms measured in them to those measured in shale, we show that the broad pore size distribution of shale can alter the appearance of capillary condensation and continuous pore filling such that they manifest much differently from the traditional IUPAC Type IV isotherm. We further demonstrate this with desorption measurements where hysteresis belies the underlying phase change. Closer scrutiny of the hysteresis loop in combination with scanning electron microscopy not only confirms the occurrence of capillary condensation but also provides evidence that just as the nanopores can alter the phase behavior of fluids, so can the nanoconfinement-induced phase behavior of the fluids impart irreversible changes to the rock, including fractures. Our observations of capillary condensation and continuous pore filling cast significant doubt upon the

\footnotetext{
* Corresponding author: ebarsott@uwyo.edu
} 
ability of simple adsorption theories to accurately model phase behavior in shale.

\section{Materials and Methods}

\subsection{Isotherm Measurements}

All isotherms were measured using a novel gravimetric apparatus. [15], [16] Unlike other systems available commercially and in the literature, our apparatus is compatible with macro-scale cores enclosed in core holders. [15] All core holders employed in this study were $3 / 4 "$ in internal diameter and 7" long and comprised a titanium body with stainless steel endcaps. During the isotherm measurements, the core holders were suspended from Mettler Toledeo XPE505C mass comparators inside of an environmental chamber (Thermotron). The environmental chamber uses air as the thermostating fluid such that experiments between 5.4 and $16.1^{\circ} \mathrm{C}$ were characterized by a temperature control of $+/-0.1^{\circ} \mathrm{C}$. In other words, the experimental fluid resided solely in the core holder, while the temperature and pressure of the thermostatic air were constant throughout all experiments. Therefore, no correction for buoyant force had to be made, [16] underscoring another unique feature of our apparatus.

By injecting or retracting the experimental fluid into or out of the core holders, its pressure could be changed, such that the change in mass and pressure at constant temperature could be used to plot an isotherm. Equilibrium was defined as the time at which the pressure of the fluid became constant as read by a Rosemount pressure transducer (Emerson). [16] At equilibrium, pressure and mass data points were generated by averaging data collected at a rate of $1 / \mathrm{sec}$ over a timespan of 1 minute. [16] Statistical analyses of these time series showed the standard error for mass and pressure to be less than $0.008 \mathrm{~g}$ and $0.008 \mathrm{psi}$, respectively, for all measurements. Because these errors are negligible, no error bars are included in the isotherm plots. We refer interested readers to our previous publications for more comprehensive discussions of the operating principle, repeatability, and accuracy of the apparatus. [15]-[17]

For adsorbents, both synthetic silica nanopores (MCM-41) and shale cores from a gas reservoir in the Middle East were used. The shale used here is from the same core that was used in our previous work. [18]

MCM-41 is a synthetic nanoporous silica popular for use in capillary condensation experiments because its singular pore size distribution and cylindrical pores simplify data analysis. Three different pore sizes of MCM-41 (Glantreo, Ltd.) were characterized using NonLocal Density Functional Theory analysis of nitrogen isotherms measured at $77 \mathrm{~K}$. [6] The characteristic pore diameters of the samples were $2.90 \mathrm{~nm}, 4.19 \mathrm{~nm}$, and 8.08 nm. [6] Henceforth, the small, medium, and large pore sizes of MCM-41 will be referred to as MCM-41-S, MCM-41-M, and MCM-41-L to be consistent with our previous work. [6] To create controlled pore size distributions, known amounts of the different adsorbents were mixed together. In one core holder, 8.2946 grams of MCM-41-S were packed. [6] In a second core holder, 4.96016 grams of MCM-41-S were mixed with 3.51795 grams of MCM-41-M. In a third core holder, 5.02250 grams of MCM-41-S were mixed with 2.31973 grams of MCM-41-L. The three different core holders allowed us to determine how large and small differences in pore size affect the shape of an isotherm.

As received shale cores were crushed by hand following the same procedure in our previous work. [18] Pieces of the crushed rock were randomly selected for characterization using a Helios 650 (ThermoFisher Scientific) focussed ion beam scanning electron microscope (FIB-SEM). Energy dispersive X-ray scattering revealed the mineralogy to comprise kerogen and calcite with minor amounts of quartz, pyrite, and illite. [18] High resolution imaging using a Through-theLens detector in downhole vision mode showed the kerogen to be highly nanoporous, as shown in Figure 1. Approximately 67 grams of the shale rock were packed into a titanium core holder for the isotherm measurement.

Propane (99\%, AirGas) was used as an adsorbate for the measurements in both types of porous media.

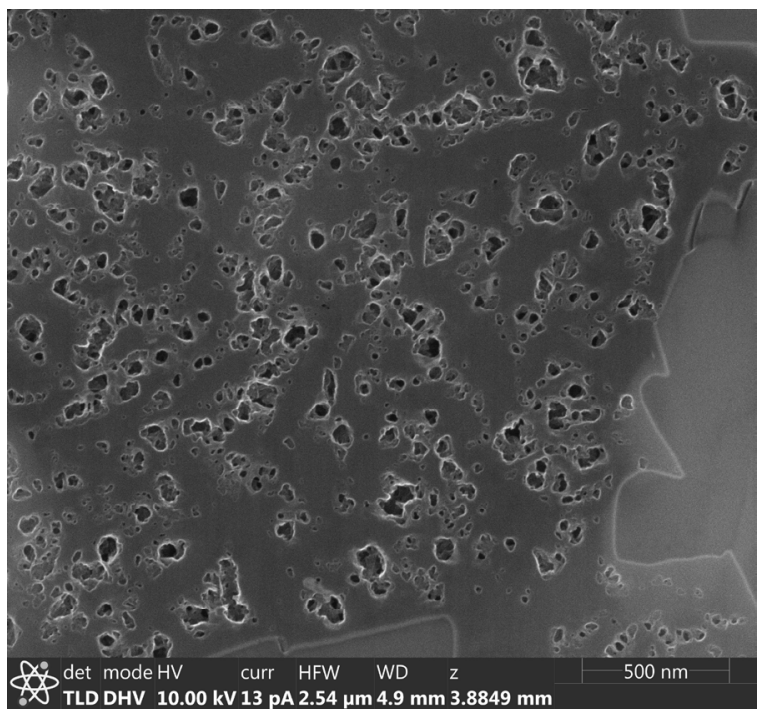

Figure 1. Scanning electron micrograph of kerogen nanopores in the shale rock taken at a voltage of $10 \mathrm{kV}$ and a current of $13 \mathrm{pA}$ with an instrument resolution of 0.8 nm.

\subsection{SEM Imaging}

One piece of the crushed shale was randomly selected for FIB-SEM imaging to determine whether the fluid phase behavior in the rock affected the rock properties. The sample was mounted on a 45-degree, pre-tilted aluminium stub (Ted Pella) using carbon tape and silver paint (Ted Pella). Then, a $20 \mu \mathrm{m}$ by $20 \mu \mathrm{m}$ cross-section of the sample was polished with the FIB-SEM's gallium ion beam. Subsequently, a $1 \mu \mathrm{m}$ diameter, $2 \mu \mathrm{m}$ deep circular hole was milled into the kerogen in the rock using 
the ion beam. Imaging of the surface was done prior to and after exposure to propane. To expose the sample to propane, it was removed from the sample stub, and placed into a pressure cell. The pressure cell was first vacuumed out using a Welch vacuum pump to remove any air and then pressurized to 6.9 bar with propane at $20^{\circ} \mathrm{C}$. After approximately $1.5 \mathrm{hrs}$, the pressure of the cell was reduced to atmospheric and the rock sample was removed, re-mounted on the 45-degree, pre-tilted stub, and imaged again. The circular hole was to determine whether any permanent changes to the kerogen structure, such as irreversible swelling, had occurred.

\section{Results and Discussion}

\subsection{Isotherms in MCM-41}

The isotherms measured in all three of the MCM-41 packs are shown in Figure 2. Isotherms were measured at $5.4^{\circ} \mathrm{C}$. Note that the bulk saturation pressure for propane at this temperature is 5.5766 bar, as reported by NIST. [19] In Figure 2, the bulk saturation pressure is measured as the rightmost abrupt increase in amount adsorbed as indicated in the plots. In all three cases, the measured bulk saturation pressure fell within $2 \%$ (percent difference) of that reported by NIST, affirming the accuracy of our measurements.

In all three isotherms, the initial increase in the amount adsorbed is propane adsorption on the pore walls. The downward concavity indicates that the propane is a wetting fluid [4] to the MCM-41.

At higher pressures capillary condensation or continuous pore filling occurs. For the pure MCM-41-S, only a single step in the isotherm is observed, indicating filling of the pores with a dense supercritical phase. Of the three pore sizes only MCM-41-L was sufficiently large to exhibit the subcritical phase transition, capillary condensation. [5] Therefore, in the mixture of MCM-41$\mathrm{S}$ and MCM-41-L, the two distinct steps in the isotherm indicate continuous pore filling for MCM-41-S and capillary condensation in MCM-41-L. When the pore sizes were closer together in the case of the mixture of MCM-41-S and MCM-41-M, the continuous pore filling in both blended together into a single step.

This has significant implications for shale rock, where the pore size distribution is broad but consecutive sizes are close together. It is often assumed that, because a distinct capillary condensation or continuous pore filling step cannot be observed in shale isotherms, neither of the nanoconfined phase phenomena is occurring. However, if we extend our understanding from the MCM-41 experiments, the absence of a step in the isotherm does not indicate the absence of capillary condensation or continuous pore filling; rather it indicates the absence of a distinct, singular pore size. In other words, phase phenomena occur gradually in the different pore sizes giving rise to a gently sloping isotherm that may be easily mistaken as an IUPAC Type I or II isotherm.

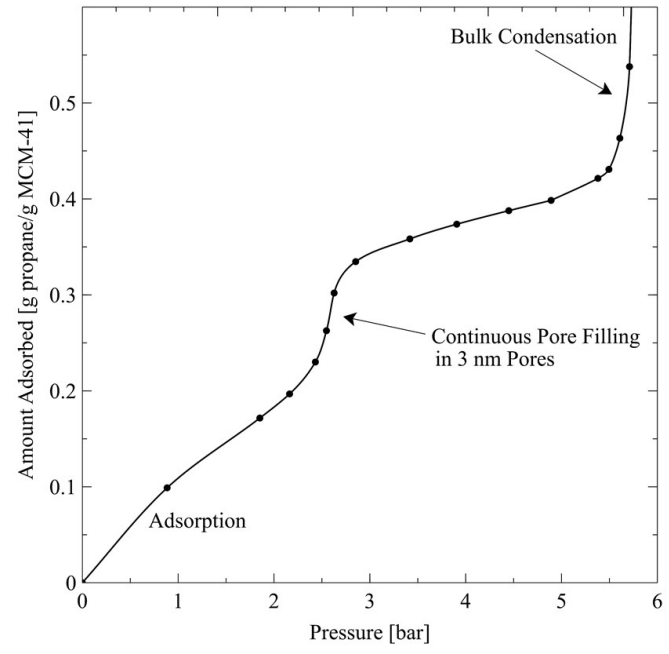

(a)

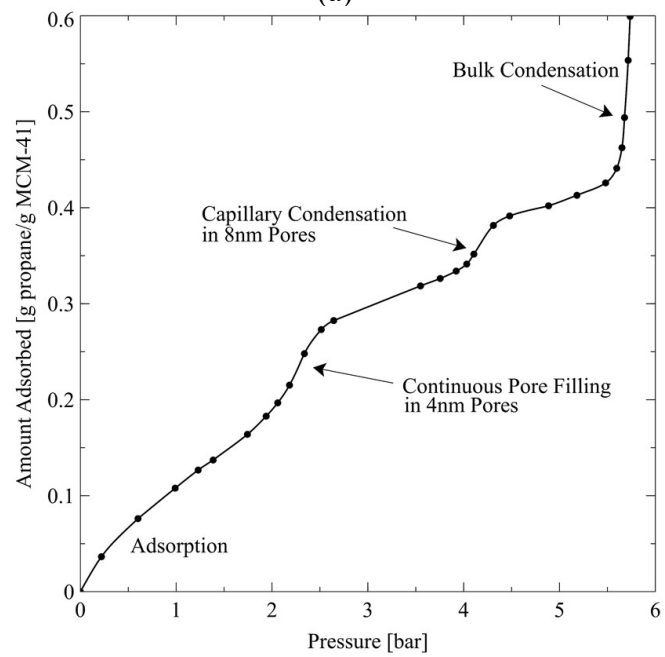

(b)

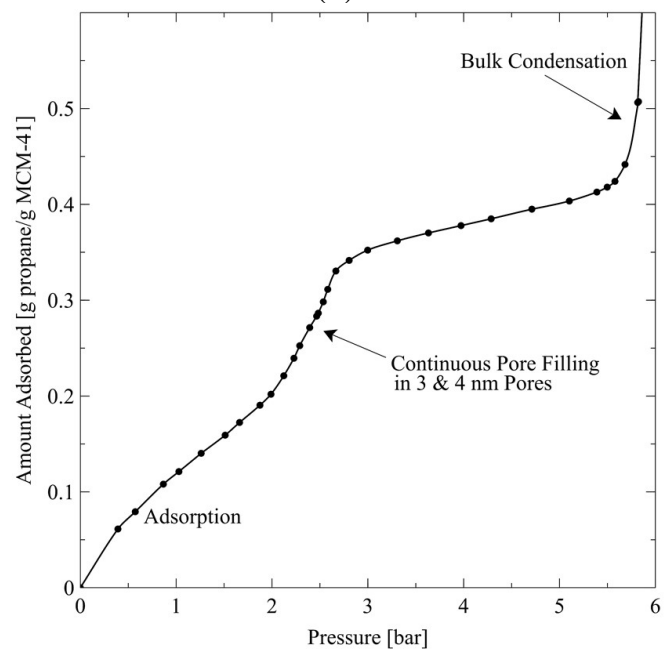

(c)

Figure 2. Propane adsorption isotherms measured at $5.4^{\circ} \mathrm{C}$ in the core holders containing (a) MCM-41-S, (b) MCM-41-S and MCM-41-L, and (c) MCM-41-S and MCM-41-M. Figure (a) adapted with permission from Barsotti et al. [6] Copyright 2018 American Chemical Society. 
Likewise, the presence of both a sub-critical and supercritical phase in the nanopores, may not only further reduce the relevance of current modeling efforts but also make accurate modeling more difficult as many equations of state are not well-equipped to work with supercritical fluids.

\subsection{Isotherms in Shale Rock}

This behavior is evident in Figure 3, where no apparent capillary condensation or continuous pore filling step is observed during adsorption, and the isotherm does, indeed, appear similar to the IUPAC Type I and II isotherms. However, the occurrence of capillary condensation in the shale sample can be verified by also measuring desorption. Upon desorption, hysteresis is observed. Hysteresis at relatively high pressures is commonly attributed to capillary condensation. [4] Therefore, because we observe hysteresis occurring at pressures as high as $90 \%$ of the bulk saturation pressure, which is approximately 7.5 bar in Figure 3, we interpret it as indicating the occurrence of capillary condensation. This interpretation is consistent with other studies in the literature where any hysteresis occurring above $50 \%$ of the bulk saturation pressure was defined as a key indicator of capillary condensation. [20] Therefore, we show that capillary condensation can occur in shale rock, even when it is not immediately apparent from the adsorption part of the isotherm.

Interestingly, the hysteresis loop for shale also appears to differ from those types classified by IUPAC. Rather than desorption occurring at larger amounts adsorbed than adsorption, [4] we observe it to occur at smaller amounts. This indicates that the fluid in the pore space is less dense during desorption than during adsorption. This can be interpreted to mean that the pore size is larger during desorption than adsorption. Thus, it may be possible not only that the shale nanopores are affecting the phase behavior of the fluid but also that the fluid can affect the morphology of the pores. In addition to our observation of hysteresis at high pressures, our observation of hysteresis at low relative pressure supports this, as it has been found in the literature to indicate adsorbent deformation. [4]

Further evidence for deformation is given by the dips observed in the isotherm at intermediate pressures. In isotherms measured for coal, these dips are often interpreted to indicate swelling of the adsorbent. [21]

To prove this, a polished cross section of the shale was visualized both before and after contact with propane at $0{ }^{\circ} \mathrm{C}$ and $\sim 6.9$ bar. Both images are presented in Figure 4. After the propane capillary condensation, large fractures were observed in the rock. Our initial hypothesis was that permanent swelling of the kerogen induced the fractures. But comparison of the $1 \mu \mathrm{m}$ diameter holes milled into the kerogen before and after propane exposure shows no change to the diameter or shape of the hole. We, therefore, hypothesize that reversible kerogen swelling

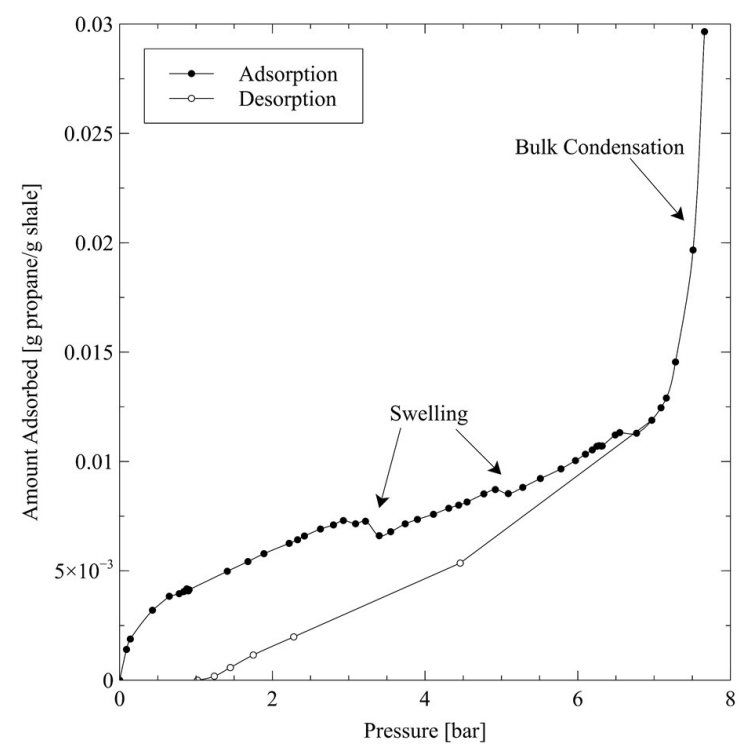

Figure 3. Propane capillary condensation isotherms measured in shale rock at $16.1^{\circ} \mathrm{C}$. Note that the bulk saturation pressure for propane at $16.1^{\circ} \mathrm{C}$ is 7.5379 bar as reported by NIST. [19] Therefore, the percent difference between our measurement and theirs is less than $1 \%$, also affirming the accuracy of this measurement.

causes fracturing. In essence, the kerogen swells during adsorption. During desorption, as the kerogen returns to its initial configuration, differences in the elasticities of the inorganic (i.e., calcite, quartz, and pyrite) and organic minerals result in fractures. Kerogen swelling has been observed previously during capillary condensation, [18], [22] but to the best of our knowledge, this is the first observation of fracturing during capillary condensation.

\section{Conclusions}

By measuring isotherms both in controlled pore size distributions of MCM-41 and shale rock, we show that complexities of the adsorbent, such as a wide pore size distribution, can cause the shape of the isotherm to appear significantly different from ideal cases presented in the literature for simple porous media. This discrepancy can lead to the misinterpretation that capillary condensation or continuous pore filling do not occur in shale. However, our observations of capillary condensation occurring gradually at different pressures across the range of pore size both in the MCM-41 and shale disprove this. Observations of hysteresis in the shale further indicate the occurrence of capillary condensation. Indeed, the complex mineralogy of shale may lead to capillary condensation-induced fractures. This indicates that the rock and fluid properties are interrelated and that current methods of PVT and core analysis that do not account for capillary condensation may lead to erroneous estimates of rock and fluid properties. The degree to which capillary condensation affects these phenomena is highly dependent upon the chemical compositions and physical morphologies of the fluid and the rock. We have proven 


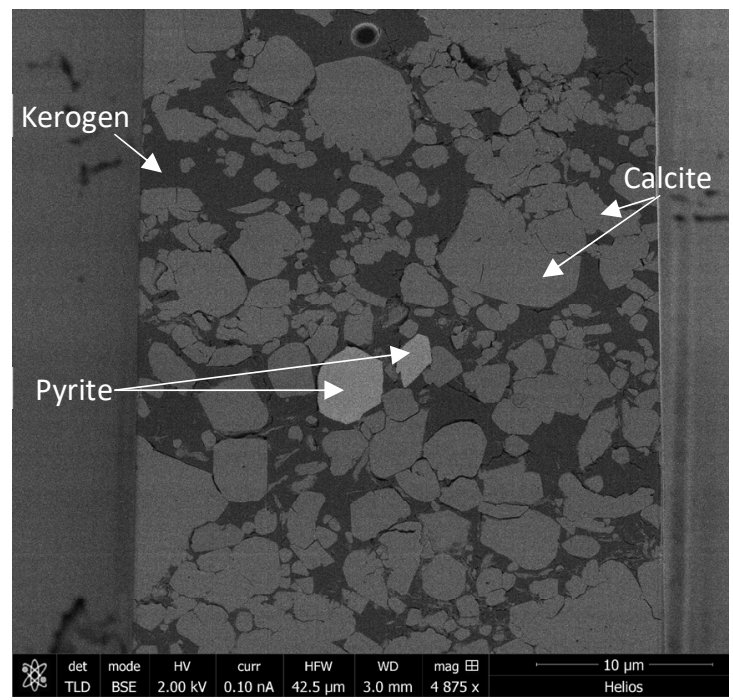

(a)

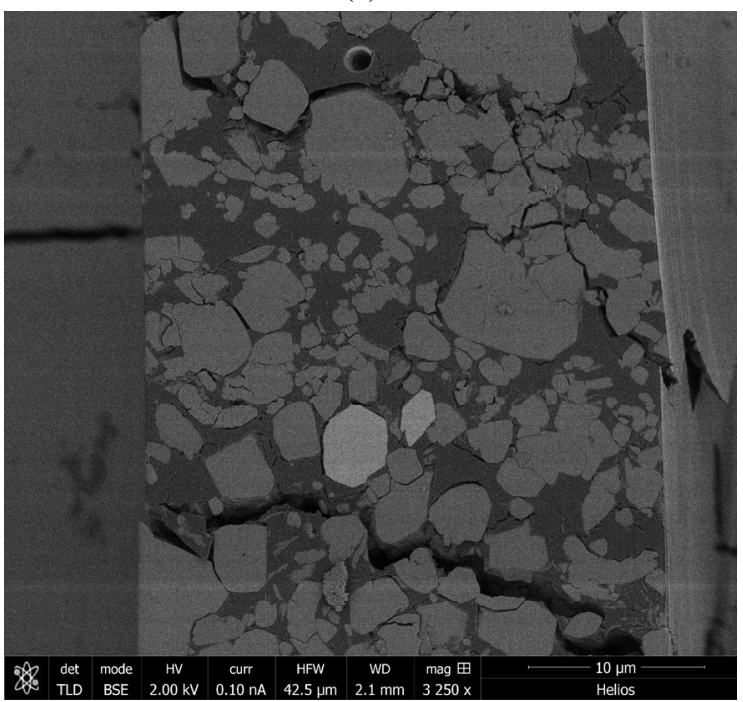

(b)

Figure 4. SEM images of a polished cross-section of the shale (a) before and (b) after exposure to propane at $20^{\circ} \mathrm{C}$ and $\sim 6.9 \mathrm{bar}$. The low, medium, and high greyscale intensity minerals are kerogen, calcite, and pyrite, respectively.

this both experimentally [16], [23] and theoretically [24] in MCM-41, where selective adsorption occurs and can affect the phase transition. Future work must include more complex mixtures in different types of reservoir cores to fully characterize this phenomenon.

We gratefully acknowledge the financial support of Saudi Aramco, Hess Corporation, and the School of Energy Resources at the University of Wyoming.

\section{References}

[1] E. Barsotti, S. P. Tan, S. Saraji, M. Piri and J.-H. Chen, "A review on capillary condensation in nanoporous media: Implications for hydrocarbon recovery from tight reservoirs," Fuel, pp. 344361, 2016.

[2] J.-H. Chen, A. Mehmani, B. Li, D. Georgi and G. Jin, "Estimation of total hydrocarbon in the presence of capillary condensation for unconventional shale reservoirs," in SPE Middle East oil and gas show and conference, Manama, Bahrain, 2013.

[3] J. Richardson and W. Yu, "Calculation of Estimated Ultimate Recovery and Recovery Factors of Shale-Gas Wells Using a Probabilistic Model of Original Gas in Place," SPE Reservoir Evaluation \& Engineering, pp. 638 - 653, 2018.

[4] M. Thommes, K. Kaneko, A. V. Neimark, J. P. Olivier, F. Rodriguez-Reinoso, J. Rouquerol and K. S. Sing, "Physisorption of gases, with special reference to the evaluation of surface area and pore size distribution (IUPAC Technical Report)," International Union of Pure and Applied Sciences \& De Gruyter, 2015.

[5] S. P. Tan, E. Barsotti and M. Piri, "Criticality of Fluids Confined in Nanopores Based on the Liquid Spinodal," Under Review, 2019.

[6] E. Barsotti, S. P. Tan, M. Piri and J.-H. Chen, "Phenomenological Study of Confined Criticality: Insights from the Capillary Condensation of Propane, n-Butane, and n-Pentane in Nanopores," Langmuir, pp. 4473-4483, 2018.

[7] S. Duan, M. Gu, X. Du and X. Xian, “Adsorption Equilibrium of $\mathrm{CO} 2$ and $\mathrm{CH} 4$ and Their Mixture on Sichuan Basin Shale," Energy \& Fuels, p. 2248-2256, 2016.

[8] S. Rani, B. K. Prusty and S. K. Pal, "Methane adsorption and pore characterization of Indian shale samples," Journal of Unconventional Oil and Gas Resources, pp. 1-10, 2015.

[9] W. Yu, K. Sepehrnoori and T. W. Patzek, "Modeling Gas Adsorption in Marcellus Shale With Langmuir and BET Isotherms," SPE Journal, pp. 589 - 600, 2016.

[10] S. Rani, E. Padmanabhan and B. K. Prusty, "Review of gas adsorption in shales for enhanced methane recovery and CO2 storage," Journal of Petroleum Science and Engineering, pp. 634-643, 2019.

[11] M. Eliebid, M. Mahmoud, S. Elkatatny, M. Abouelresh and R. Shawabkeh, "Adsorption Role in Shale Gas Recovery and the Feasibility of $\mathrm{CO} 2$ in Shale Enhanced Gas Recovery: A Study on Shale Gas from Saudi Arabia," in SPE Kuwait Oil $\&$ Gas Show and Conference, Kuwait City, Kuwait, 2017. 
[12] V. Nguyen-Le and H. Shin, "Development of reservoir economic indicator for Barnett Shale gas potential evaluation based on the reservoir and hydraulic fracturing parameters," Journal of Natural Gas Science and Engineering, pp. 159167, 2019.

[13] S. Yang, K. Wu, J. Xu, J. Li and Z. Chen, "Roles of multicomponent adsorption and geomechanics in the development of an Eagle Ford shale condensate reservoir," Fuel, pp. 710-718, 2019.

[14] H. Belyadi and M. Smith, "A Fast-Paced Workflow for Well Spacing and Completions Design Optimization in Unconventional Reservoirs," in SPE/AAPG Eastern Regional Meeting, Pittsburgh, Pennsylvania, USA, 7-11 October 2018.

[15] E. Barsotti, S. Saraji and M. Piri, "Nanocondensation Apparatus". United States of America Patent US15/588,094, 2017.

[16] E. Barsotti, S. Saraji, S. P. Tan and M. Piri, "Capillary Condensation of Binary and Ternary Mixtures of n-Pentane-Isopentane- $\mathrm{CO} 2$ in Nanopores: An Experimental Study on the Effects of Composition and Equilibrium," Langmuir, p. 1967-1980, 2018.

[17] E. Barsotti, S. P. Tan, M. Piri and J.-H. Chen, "Capillary-Condensation Hysteresis in NaturallyOccurring Nanoporous Media," Under Review, 2019.

[18] E. Barsotti, S. P. Tan, M. Piri and J.-H. Chen, "Capillary-Condensation Hysteresis in NaturallyOccurring Nanoporous Media," Under Review, 2019.

[19] E. Lemmon, M. McLinden and D. Friend, NIST Standard Reference Database Number 69, P. Linstrom and W. Mallard, Eds.

[20] H. Bu, Y. Ju, J. Tan, G. Wang and X. Li, "Fractal characteristics of pores in non-marine shales from the Huainan coalfield, eastern China," Journal of Natural Gas Science and Engineering, vol. 24, pp. 166-177, 2015.

[21] R. Pini, S. Ottiger, G. Storti and M. Mazzotti, "Role of Adsorption and Swelling on the Dynamics of Gas Injection in Coal," Journal of Geophysical Research Solid Earth, vol. 114, no. 84, 2009.

[22] M. Pathak, H. Kweon, M. Deo and H. Huang, "Kerogen Swelling and Confinement: Its implication on Fluid Thermodynamic Properties in Shales," Scientific Reports, vol. 7, 2017.

[23] E. Barsotti, M. Piri, J.-H. Chen and S. Althaus, "Solution Gas Drive in Tight Oil Reservoirs: New Insights from Capillary Evaporation Experiments," in $S P E / A A P G / S E G$ Unconventional Resources Technology Conference, Houston, Texas, USA, 2018.

[24] S. P. Tan, E. Barsotti and M. Piri, “Application of material balance for the phase transition of fluid mixtures confined in nanopores," Fluid Phase Equilibria, 2019. 